


\section{SIEMENS- HANDBÜCHER}

Herausgegeben von der Siemens \& Halske A.-G. und den Siemens-Schuckertwerken, G. m. b. H.

\section{Band: \\ Das Kraftwerk Fortuna II}

\footnotetext{
WALTER DE GRUYTER \& CO. BERLIN UND LEIPZIG 1925
} 


\section{DAS KRAFTWERK FORTUNA II}

\section{MONOGRAPHIE \\ EINES DAMPFKRAFTWERKS IN SYSTEMATISCHER DARSTELLUNG}

vON

ALBERT SCHREIBER

DIREKTOR DES RHEINISCHEN ELEKTRICITATSWERKS

IM BRAUNKOHLENREVIER A. G., KOLN

MIT 141 ABBILDUNGEN IM TEXT UND AUF 7 TAFELN

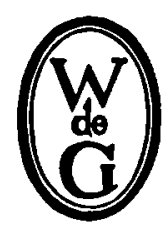

WALTER DE GRUYTER \& CO.

BERLIN UND LEIPZIG

1925 
Alle Rechte, insbesondere das der Ubersetzung, vorbehalten

Copyright by Walter de Gruyter \& Co, Berlin and Leipzig 1925 